\title{
In situ observations of low-density regions inside the plasmasphere
}

\author{
G. Kotova ${ }^{1}$, V. Bezrukikh ${ }^{1}$, M. Verigin ${ }^{1}$, and J. Smilauer ${ }^{2}$ \\ ${ }^{1}$ Space Research Institute, Russian Academy of Sciences, Moscow 117997, Russia \\ ${ }^{2}$ Institute of Atmosphere Physics, Czech Academy of Sciences, Prague, Czech Republic \\ (Received January 30, 2004; Revised April 19, 2004; Accepted September 24, 2004)
}

\begin{abstract}
Thermal plasma measurements performed on MAGION 5 (subsatellite of INTERBALL 1) in the plasmasphere of the Earth are analyzed in conjunction with simultaneous solar wind data and ground-based ionospheric measurements in quiet geomagnetic conditions, during and after geomagnetic storms. In situ satellite observations reveal the existence of long-lived (2-3 days) depleted regions (MLT width $\gtrsim 1.5-2$ hours) in the plasmasphere that extend out from $L \sim 3$. These observations well correspond to 'notch' or 'bite-out' regions found by the IMAGE spacecraft. Possible reasons for the formation of low-density regions are discussed.
\end{abstract}

Key words: Plasmasphere, plasmapause, thermal plasma, geomagnetic activity.

\section{Introduction}

Complicated morphology of the outer regions of the Earth's plasmasphere was experimentally observed and theoretically investigated since the plasmapause and plasmasphere discovery. The average plasmapause position was analyzed as a function of geomagnetic activity (Carpenter and Park, 1973; Carpenter and Anderson, 1992; Moldwin et al., 2002), though it was pointed out on the complexity of the boundary and its dynamics. For example, difference in plasmapause $L$ value $(\Delta L \sim 1)$ was observed by the VLF/OGO 4 data near 05 LT between 2 successive orbits of the satellite within $\sim 20$ degrees in longitude (Carpenter and Park, 1973; Carpenter and Chappell, 1973). It was interpreted in terms of the rotation under the satellite of the structures formed in earlier local time sectors (around midnight) under the action of the inferred unsteady electric fields during the substorm. A complete review of plasmasphere studies during approximately 35 years was given by Lemaire and Gringauz (1998).

In the first experiments there were already seen troughs in the density versus $L$-shell profiles followed by density increases before the plasmapause crossing (Carpenter et al., 2000, and references there in). Horwitz et al. (1990) classified plasmaspheric profiles inward of the plasmapause observed by the retarding ion mass spectrometer (RIMS) on DE 1. Six types of density profiles were marked depending on the number and locations of troughs and plateaus relative to the plasmapause. Regions of high density plasmaspheric plasma have been observed across the dayside magnetosphere by geostationary satellites (e.g., GEOS 2, Higel and $\mathrm{Wu}, 1984)$. Troughs in plasmaspheric density profiles were also observed by the ALPHA 3/INTERBALL 1 experiment when the satellite crossed the dusk plasmasphere almost perpendicularly to the equatorial plane. The troughs were seen

Copy right (c) The Society of Geomagnetism and Earth, Planetary and Space Sciences (SGEPSS); The Seismological Society of Japan; The Volcanological Society of Japan; The Geodetic Society of Japan; The Japanese Society for Planetary Sciences; TERRAPUB. on the same $L$-shells in the southern and northern hemispheres, thus suggesting that the whole plasmaspheric flux tube was empty and located closer to the Earth than filled flux tube (Kotova et al., 2002a). Unfortunately, in situ spacecraft as well as whistler observations do not permit to distinguish the isolated plasma regions from more complicated formations which may really be a prolongation of the plasmasphere somewhere connected to its main body. Plasma regions seen as isolated were interpreted both as detached clouds (Chappell, 1974) and as filamentary extensions of the dusk plasmasphere bulge (plasma tails) (Chen and Grebowsky, 1974). An alternative mechanism of plasma tail formation which fits currently available observations was developed by Lemaire (2000).

Analyzing in situ or whistler observations it is not also easy to distinguish troughs inside the plasmasphere from the depleted regions narrow in longitude but extending outward to the plasmapause as it observes in the neighbor parts of the plasmasphere. These later plasmasphere features were revealed by Extreme Ultraviolet Imager aboard the IMAGE spacecraft. They were called 'voids', 'bite-outs' and finally 'notches' (Sandel et al., 2001; Sandel et al., 2003).

In the present paper in situ observations of localized lowdensity regions of the plasmasphere with the MAGION 5 subsatellite of INTERBALL 2 will be considered.

\section{MAGION 5 Observations}

Retarding plasma analyzer (PL-48) for cold ion flux measurements was continuously functioning on the auroral satellite MAGION 5. Energy spectra were measured during 0.4 $\mathrm{s}$ once per $\sim 8 \mathrm{~s}$ (The description of the instrument can be found in Bezrukikh et al., 1998). The orbit perigee was about $1.2 R_{E}$ (Earth redius), and apogee- $\sim 4 R_{E}$. The orbital period of MAGION 5 was $\sim 6$ hours, but due to different reasons the data tended only occasionally to be available on 2 successive plasmasphere passes (mainly inbound) per day. Parts of Magion 5 trajectories where the data were acquired are presented in Fig. 1. 



3 Jan. 2001, 14.10-15.20 UT

Fig. 1. Examples of MAGION 5 inbound trajectory parts in the inner magnetosphere. Meridian (upper) and equatorial (lower) projections. The axes are scaled by the Earth's radius $\left(R_{E}\right)$.

Figure 2(a) presents observations in the pre midnight sector on October 27-November 2, 2000. Two columns show the data obtained from 2 successive orbits per day with 6 hour difference. In each column the graphs referred to measurements approximately with 24 hour difference and thus related to almost the same region in the plasmasphere. Variations of magnetic activity indices during the period of observations are shown in Fig. 2(b). In the left column in Fig. 2(a) the first profile after the storm (No. 3) shows well depleted outer plasmasphere but a 'bulge' was observed at $L \sim 3.2$. This 'bulge' disappeared in the profile No. 3a, obtained 6 hours later. In a day fully depleted outer plasmasphere was observed, while again 6 hours later almost 'normal' plasmasphere profile was recorded. Unfortunately profile No. 5 was missed, but on the day after, the situation repeated. Thus, after the storm for probably at least 3 days the plasmasphere was depleted in the confined corotating region at $L>3$ as observed in the left set of observations. The right set of observations shifted by 6 hours shows filled plasmasphere. Assuming the depleted regions to corotate with the Earth, the longitude extension of such region can be estimated as not less than 1.5-2 hours (note the difference in UT for profiles
No. 4 and 6). This feature in the plasmasphere seems similar to low-density regions ('notches') detected by the IMAGE spacecraft. We tried to check the ionospheric stations (http://spidr.ngdc.noaa.gov/spidr/) more or less correspondent to the location of corotating depleted region Salekhard ( $L=4.5$, delay in magnetic local time relative to zero magnetic meridian $\triangle \mathrm{MLT}=4.8)$ and Loparskaya $(L=5.3$, $\triangle \mathrm{MLT}=2.9$ ). It was found that above Salekhard on October 29-November 2 and Loparskaya on October 29November 1 F2 layer critical frequency and hence the density in the ionospheric F2 layer is depressed especially during the daytime. For comparison, St. Petersburg $(L=3.2$, $\Delta \mathrm{MLT}=2.5$ ) data show only usual storm time critical frequency depletion on October 29-30.

Another example of low-density region observed by Magion 5 is shown in Fig. 3(a). The probable 'notch' region is seen in panels 3. Again this phenomenon is observed after a small magnetic storm on September 12 (Fig. 3(b)). Figure 3(a) shows that during small storm intensification the plasmapause (where thermal plasma density drops by a factor of $>5$ to values less than $20 \mathrm{~cm}^{-3}$ ) approached the Earth and inside the plasmasphere the density increased $(2,5,5 a)$ 
MAGION 5 October - November $2000 \quad 22.0$ - 23.3 MLT

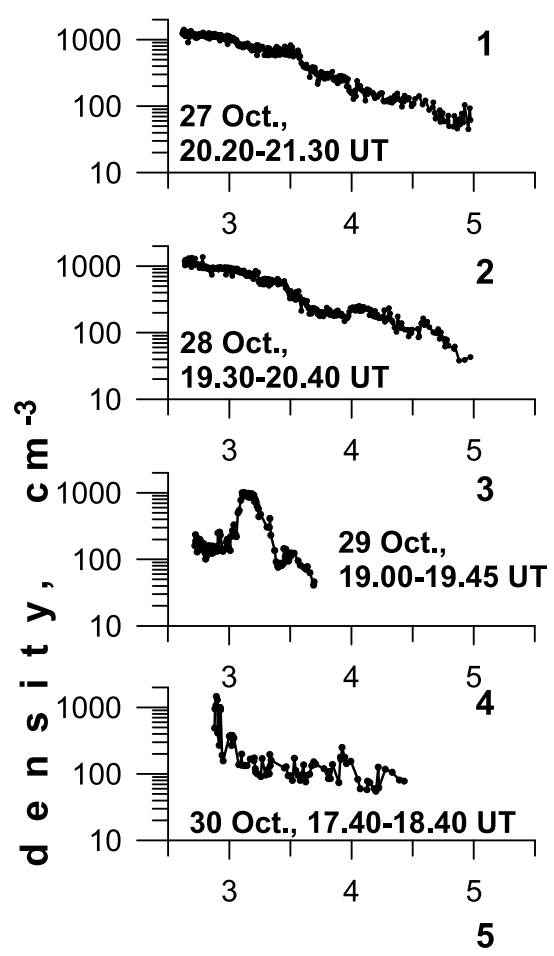

$1 \mathbf{a}$

no data



no data

$2 a$

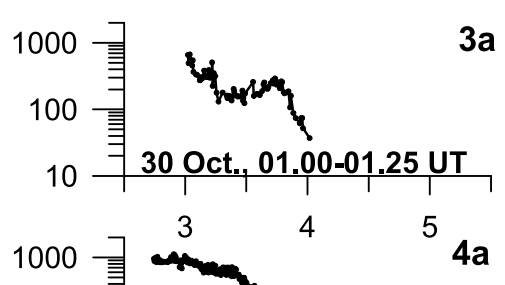

$3 \mathbf{a}$



no data
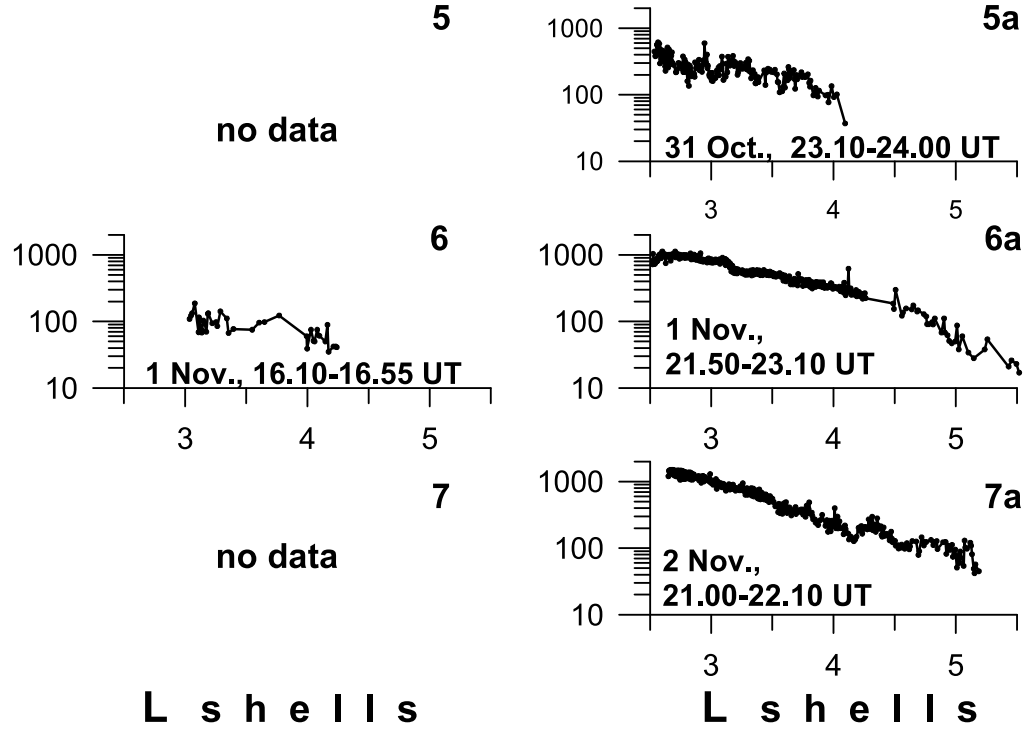

L s h e I I s

Fig. 2(a). Density versus $L$ profiles measured at 22.0-23.3 MLT in a number of successive inbound plasmasphere passes during 27 October-2 November 2000.

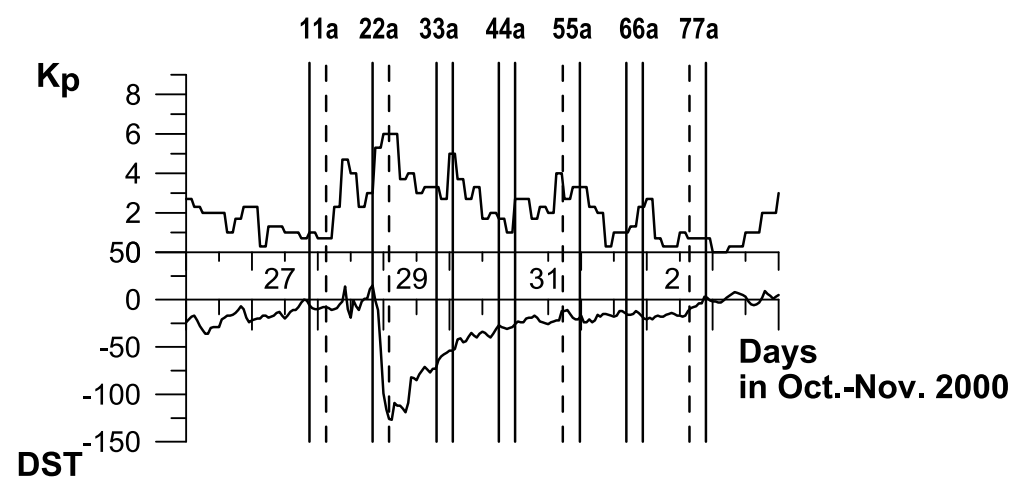

Fig. 2(b). Variations of Kp and Dst indices during 27 October-2 November 2000. Vertical solid lines mark the times of measurements for profiles shown in Fig. 2. (Dashed lines mark the times of orbits when no data were obtained.) 


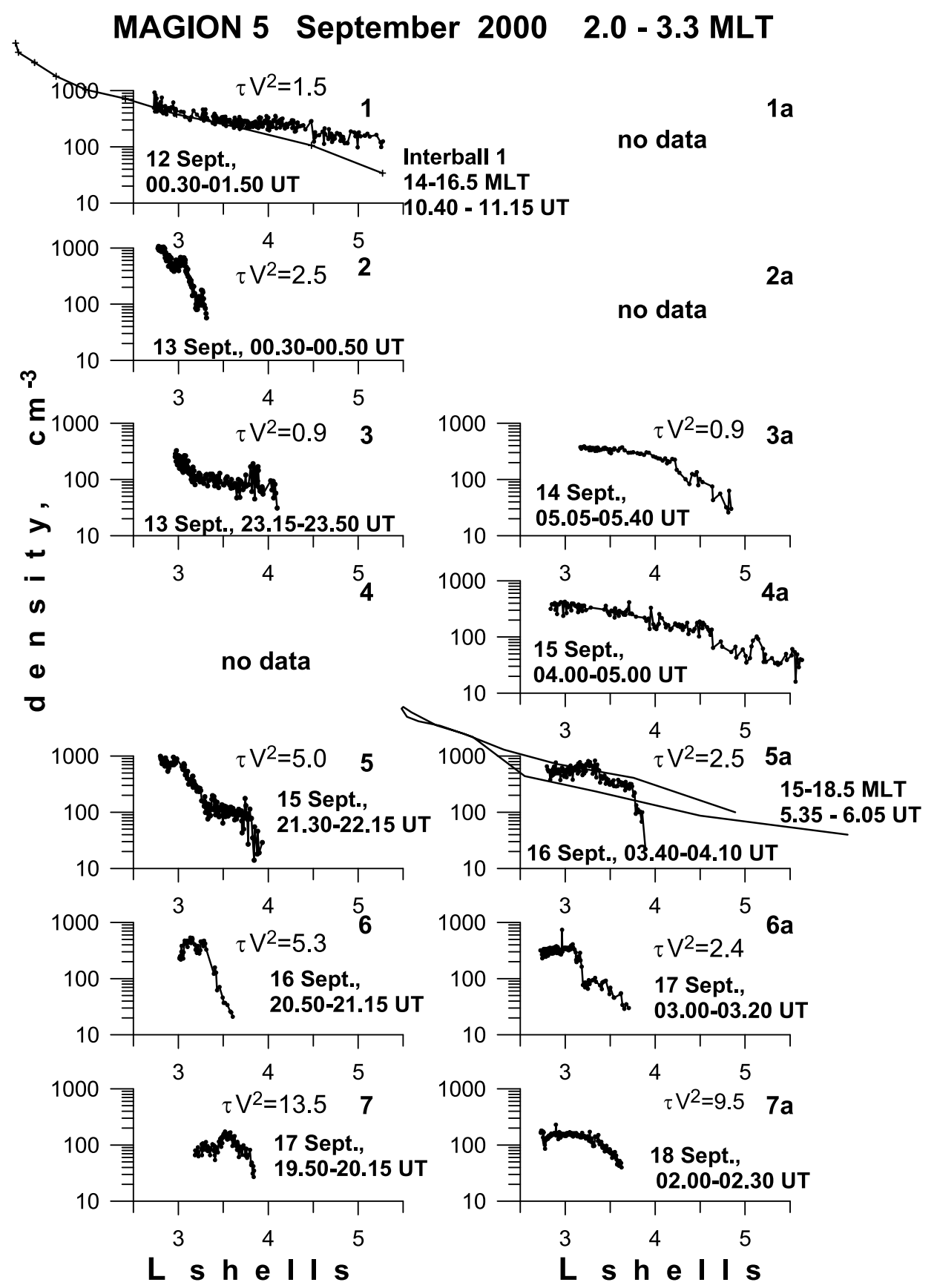

Fig. 3(a). Density versus $L$ profiles measured at 2.0-3.3 MLT in a number of successive inbound plasmasphere passes during 12-18 September 2000. $\rho V^{2}$ values give the solar wind dynamic pressure measured by the ACE spacecraft and shifted to the Earth (http://cdaweb.gsfc.nasa.gov/). In panels 1 and 5 a Interball 1 measurements are shown for comparison.



Fig. 3(b). Variations of Kp and Dst indices during 12-18 September 2000. Vertical solid lines mark the times of measurements for profiles shown in Fig. 2. (Dashed lines mark the times of orbits when no data were obtained.) 

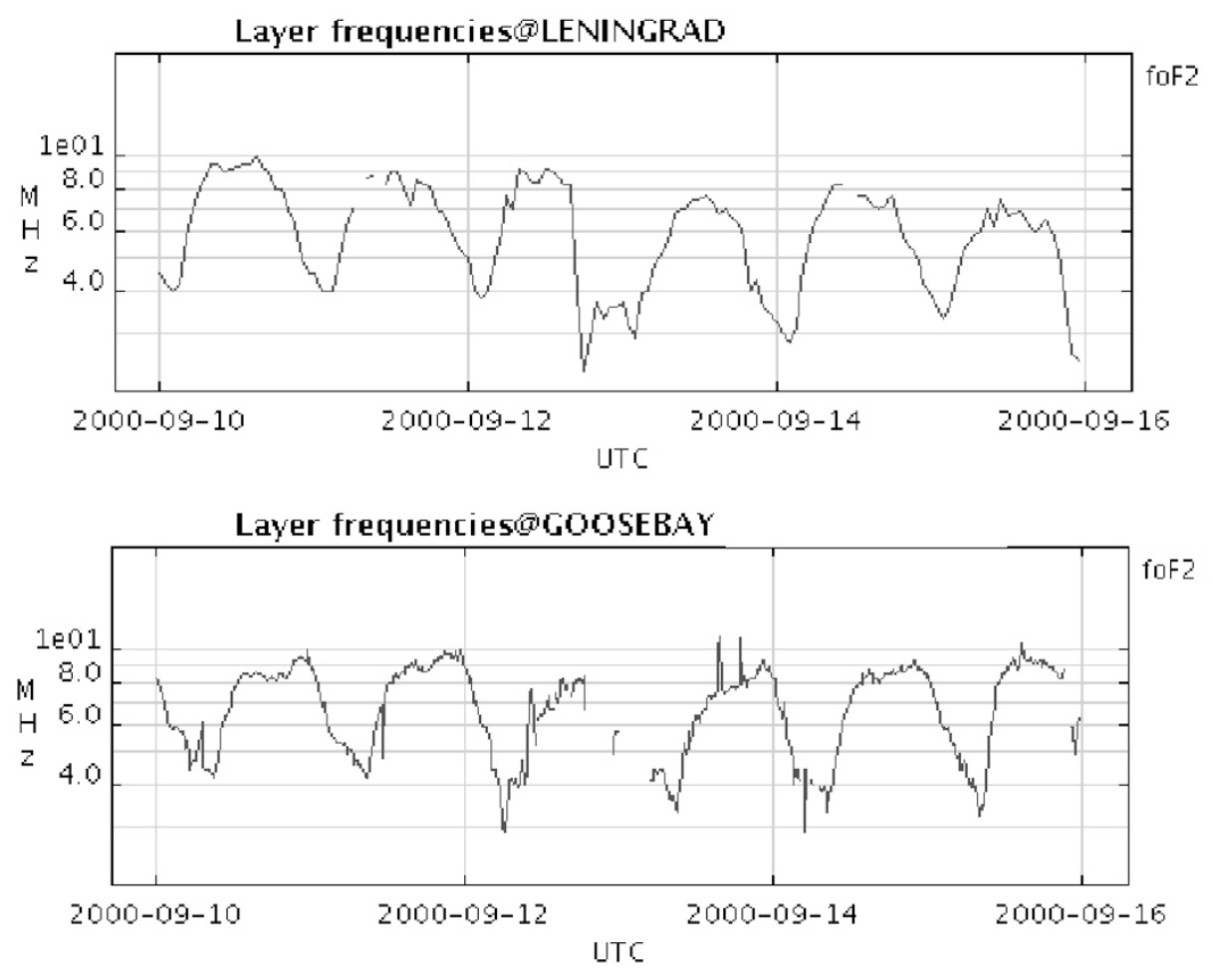

Fig. 4. Critical frequencies of ionospheric F2 layer provided by http://spidr.ngdc.noaa.gov/spidr/.

while it is generally accepted that the plasmasphere density is depressed in response to magnetic storms. This could also be a consequence of the enhanced solar wind dynamic pressure $\left(\rho V^{2}\right)$ during plasmasphere crossings shown in Fig. 3(a)panels 2, 5, 5a. (ACE data at http://cdaweb.gsfc.nasa.gov/ were shifted to the Earth's position; proton ram pressure was used as a proxy of the solar wind ram pressure). INTERBALL 1 measurements previously revealed that cold ion density in the outer plasmasphere $(L>2.8)$ increases with increasing solar wind ram pressure (Kotova et al., 2002a, b). The successive strong storm intensification on 17 September resulted in significant decreasing of thermal plasma density $(7,7 \mathrm{a})$. In panels 1 and 5a the INTERBALL 1 data obtained on the dusk side 10 and 2 hours later, respectively, are given for comparison. It is seen that on the dusk side the density was similar, but after the small storm on September 16 the plasmasphere extended much farther from the Earth (5a). Rather small storm did not affect the dusk plasmasphere, the INTERBALL 1 observations referred to September 8, 12, and 16 (this satellite has the orbital period of $\sim 4$ days) show practically identical density vs. $L$ profiles.

Panel 3 in Fig. 3(a) nearly corresponds to observations above St. Petersburg $(L=3.2, \triangle \mathrm{MLT}=2.5)$ and panel $3 \mathrm{a}$ to observations above Goozebay ( $L=$ 4.4, $\Delta$ MLT $=20.9$ ). Ionospheric data provided by http://spidr.ngdc.noaa.gov/spidr/ are shown in Fig. 4. The density in the F2-layer of the ionosphere is proportional to the foF2 frequency squared. Above St. Petersburg on September 10-12 maximum critical frequencies reached 9$10 \mathrm{MHz}$ and on September 13-15 foF2 was in the range 7.4$8.2 \mathrm{MHz}$ in the daytime. Hence, the maximum daytime ionospheric density is 1.5-2 times reduced above St. Petersburg (Leningrad) on September 13-15 and practically no effect is seen in Goosebay data. While obviously ionospheric density corresponds to oxygen density mainly and the plasmasphere is filled mostly by hydrogen, the whole flux tube might be depleted.

Figure 5 again shows the evolution of low-density region inside the plasmasphere observed on the dusk side in January 2001. For comparison dashed profiles in panels $2 \mathrm{a}, 3 \mathrm{a}$, and 4a represent the profiles in panels 2, 3, 4, respectively. After a weak storm the depleted region is clearly seen in panels 22a and 3-3a. This depletion disappeared in $\sim 2$ days after the storm, and the plasmasphere density profiles observed with 6 hour difference are very similar, that is clearly seen from the superposition in panel 4a. This time low plasmaspheric density probably corresponds to low solar wind density (ACE data at http://cdaweb.gsfc.nasa.gov/). No ionospheric effect was found in this case probably because the closest station in St. Petersburg is still not in proper MLT sector.

\section{Comparison with IMAGE Results and Discus- sion}

Figure 6(a) gives an example of notch observation by the Image EUV instrument on 24 June 2000. The EUV instrument measures the radiation at $30.4 \mathrm{~nm}$ and thus the plot brightness is proportional to the line integral of the $\mathrm{He}^{+}$ abundance along lines of sight. Owing to the rapid drop in plasma density with increasing $L$, the brightness is mainly corresponds to $\mathrm{He}^{+}$density at minimum $L$ along the line of sight. The image in Fig. 6(a) was obtained from high northern latitudes and the line of sight is nearly perpendicular to the equatorial plane, so the brightness is connected to the equatorial plasma density.

In Fig. 6(a) the sun is to the right and the Earth's shadow is to the left. A prominent low-density region or "notch" 
MAGION 5 January $2001 \quad 15.7-17.5$ MLT

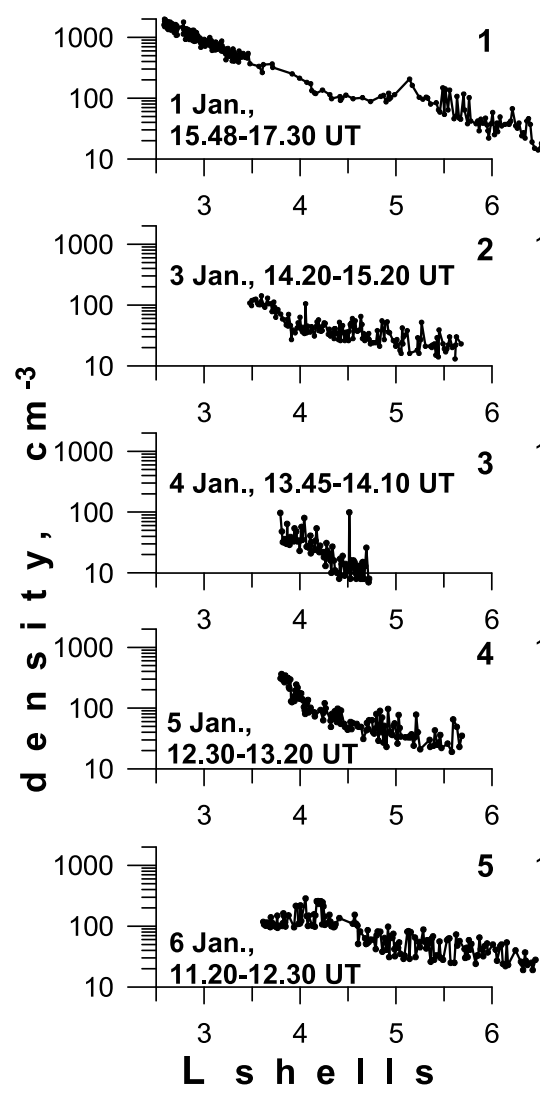

$1 \mathrm{a}$

no data

$\beth^{3}$ Jan., 20.30-21.00 UT $2 \mathrm{a}$
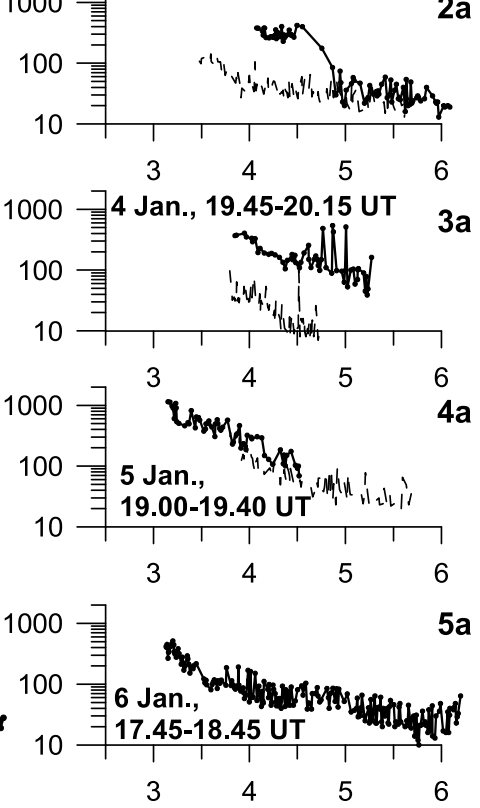

1

$22 a 33 a 44 a 55 a$

L s h e I I s


Fig. 5. Density versus $L$ profiles measured at 15.7-17.5 MLT in successive inbound plasmasphere passes during 1-6 January 2001. Botom plots present $\mathrm{Kp}$ and Dst variations during the same time period, $\rho V^{2}$ values give the solar wind dynamic pressure measured by the ACE spacecraft and shifted to the Earth (http://cdaweb.gsfc.nasa.gov/). Dashed profiles in panels $2 \mathrm{a}, 3 \mathrm{a}$, and 4a repeat the profiles in panels $2,3,4$ respectively.

is seen in the lower left part of the image. This notch was observed co-rotating with the magnetic field during 41 hours. "A typical notch has an azimuthal extent of $5^{\circ}$ to $20^{\circ}$, a radial extent of 1 to $2.5 R_{E}$, and a brightness contrast of a factor of $\sim 3$ against the main body of the plasmasphere" (Sandel et al., 2003). In Figs. 2(a), 3(a) MAGION 5 data show density reduction from $L \sim 3$ (it is worth noting that there are no MAGION 5 data inward of $L \sim 2.5$ ) and at $L \sim 3.5$ the density is reduced by a factor of 3-4 compared to the closest plasmasphere crossing. Figure 5 shows deeper plasmasphere depletion (by a factor of 5-8) but in the outer region. The radial extent of the depleted regions observed by MAGION 5 is also quite similar to that of notches. The azimuthal extent of MAGION 5 structures is probably larger than typical extent of notches but it is obviously hard to observe narrow structures by in situ methods.

Sandel et al. (2001) pointed that notches are most often observed during times of geomagnetic quiet. However the notch shown in Fig. 6(a) was really observed on the quiet day of 24 June 2000 but the previous day was rather disturbed with 2 AE bursts approximately at time when the proper corotating plasmasphere region intersected the nighttime sector (Figs. 6(b) and (c)). Hence, it is possible that the low-density region is a consequence of magnetic activity that affected the limited region in the plasmasphere.

The authors of the IMAGE EUV experiment suggest that observed low intensity regions correspond to reduced density in a complete flux tube extending from the equator to the ionosphere. The notch shown in Fig. 6(a) was observed at $\sim 3.00$ MLT that roughly corresponds to Yakutsk location $(L=3.15, \triangle \mathrm{MLT}=8.7)$. Really, measurements by Yakutsk ground-based station (A. E. Stepanov, private communication) show reduced plasma frequency corresponding to ionospheric density in the F2 layer $\left(N_{e} \sim(\text { foF } 2)^{2}\right)$ during 




Fig. 6(a). EUV image showing the distribution of $\mathrm{He}^{+}$in the Earth's plasmasphere obtained at 17.12 UT on 24 June 2000. The sun is to the right. Big arrow marks the notch region, small arrow pointed to the shoulder.

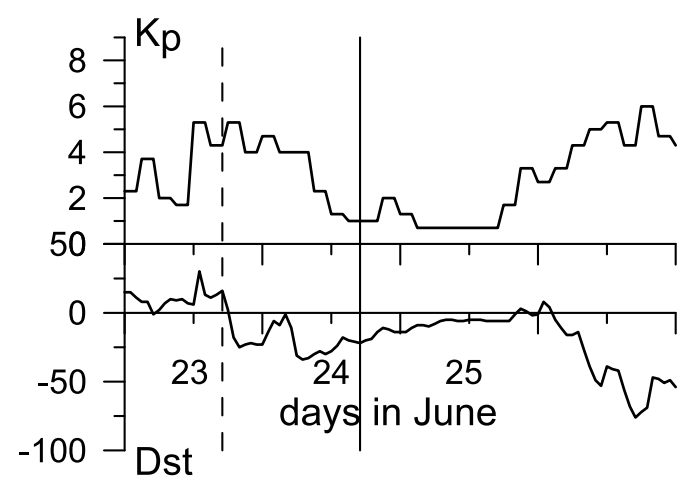

Fig. 6(b). Variations of Kp and Dst indices during 23-26 June 2000. Vertical solid line marks the times of IMAGE measurements shown in Fig. 3. Dashed line marks the same hour a day before.

at least a day after the observed frequency trough associated with magnetic disturbances on June 23. It is likely that reduced density in a complete flux tube including ionosphere resulted in slow refilling of the tube, and thus the notch phenomenon is observed during 2-3 days without significant changing.

IMAGE also observed shoulder-like bulges with sharp eastern edge about $0.5 R_{E}$ in radial extent that gradually tapered off on the west side (Fig. 6(a)). Generally speaking it is impossible to exclude fully the possibility that considered Magion 5 data refer not to observations of 'notches' but to 'shoulders'. However, observations in Figs. 2 and 3 most likely corresponds to 'notch' since the position of the inner abrupt density drop before the 'depleted region' (left panels) is at least more than $1 R_{E}$ within the 'normal' plasmasphere (right panels). Panels 5-5a in Fig. 3(a) might correspond to 'shoulder' observation.

Unfortunately we did not succeed to find simultaneous

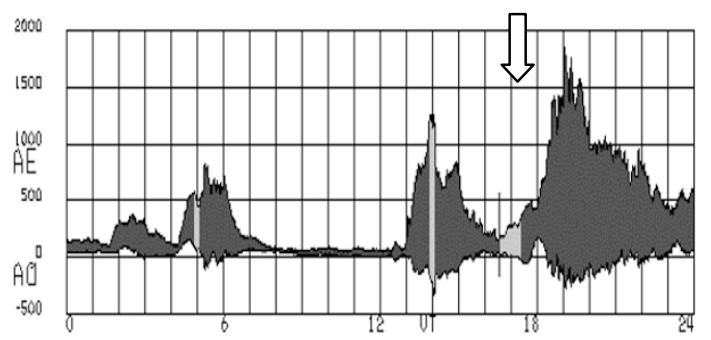

Fig. 6(c). Variations of AE and AQ indices on 23 June 2000. The arrow marks the time a day before the IMAGE measurements shown in Fig. 3.

\section{MAGION 5 April 2001}

8.3 - 9.1 MLT
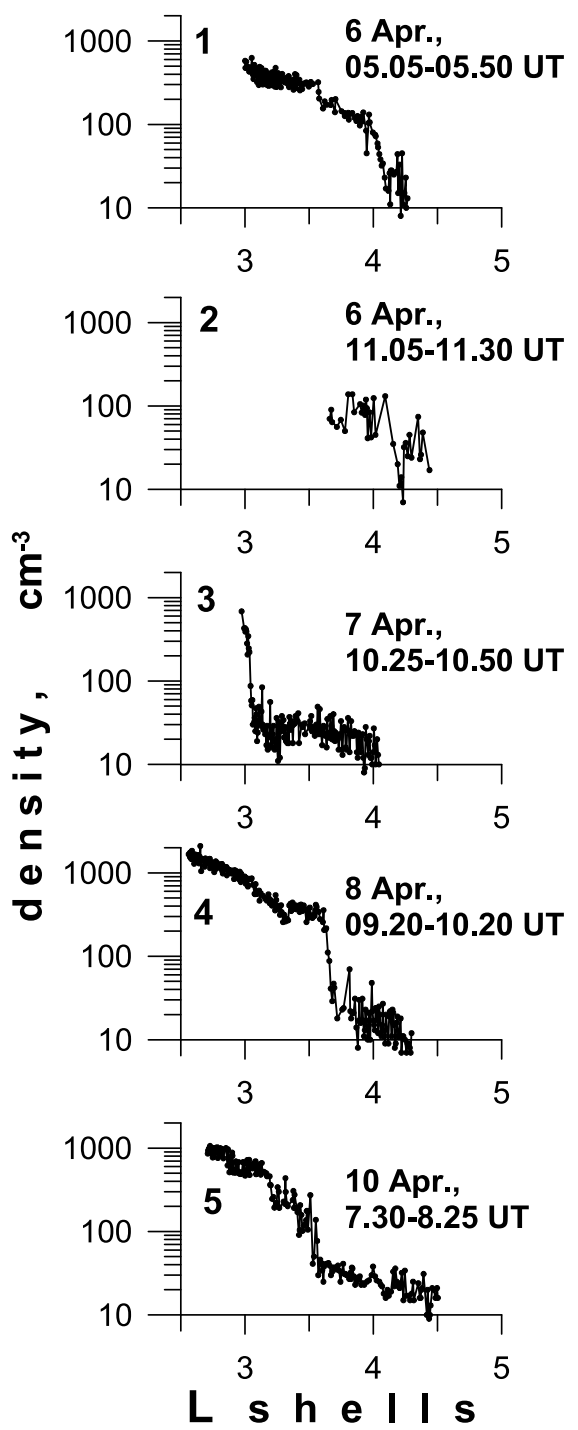

Fig. 7. Density versus $L$ profiles measured at 8.3-9.1 MLT in a number of successive inbound plasmasphere passes in April 2001.

clear notch observations by the MAGION 5 and IMAGE satellites. Probably MAGION 5 data obtained in April 2001 (Fig. 7), correspond to IMAGE observations on 7 April, 2001 23.52 UT considered by Green et al. (2002) and presented 
in their Fig. 3. We have MAGION 5 data from only one plasmasphere crossing per day at that time. The data in panel 3 obtained on 7 April, 10.25-10.50 UT in the dawn plasmasphere is possibly referred to $\sim 11$ hours preceding IMAGE observation of notch region in the evening sector (not that one analyzed in details by Green et al. (2002)).

In the past, sometimes variations in plasmapause $L$ value $(\Delta L \sim 1)$ between 2 successive orbits of the satellite were detected (e.g., OGO 4 data, Carpenter and Chappell, 1973), though not as a quasi stable phenomenon observed during 23 days. Plasmapause excursions toward the Earth, detected close to 05 MLT, were explained by the rotation under the satellite of the structures formed in earlier local time sectors (around midnight) under the action of the inferred unsteady electric fields during the substorm. Even earlier whistler research (Carpenter et al., 1972) found that during weak to moderate magnetic storms enhanced cross $-L$ inward drifts close to plasmapause occur preferentially in the 2300-0200 MLT sector and may sometimes occur in a wider region extending to local dawn. Above analyzed MAGION 5 data suggest that these inward drifts possibly result in formation of depleted regions-notches-in the plasmasphere. Published IMAGE data also do not contradict to this suggestion.

So far the only explanation has been proposed for the formation of notches by Pierrard and Lemaire (2004). It is based on the plasmapause definition as "the innermost convection contour tangent to the Zero Parallel Force surface where the field-aligned components of the gravitational force and of the centrifugal force balance each other". The authors use an empirical Kp-dependent electric field model of McIlwain (1986). Pierrard and Lemaire suggest that formation of a notch is associated with a fast increase and decrease of $\mathrm{Kp}$. The above considered observations were really correlated with some magnetic activity, however to verify this mechanism it is necessary to check whether all the available observations of notches are accompanied by proper Kp variations. In all likelihood formation of notches is connected to the enhanced radial and/or azimuthal electric field in a limited region of the magnetosphere. Thus to determine a mechanism responsible for notch formation the simultaneous measurements of plasma structure and electric field are necessary.

\section{Conclusion}

In situ satellite observations reveal the existence of longlived ( $2-3$ days) depleted regions ( $\triangle$ MLT $\gtrsim 1.5-2$ hours) in the plasmasphere that extend out from $L \sim 3$. These observations well correspond to the observations of 'notches' by the IMAGE spacecraft.

Simultaneous ionospheric ground-based data show depletions in the daytime F2 layer density at the feet of 'notch' regions.

Magion 5 observations suggest that formation of lowdensity regions is a consequence of magnetic activity that affected the limited section of the plasmasphere.

Acknowledgments. This work was performed under RFBI grant 04-02-16666-a and also was supported by grant A3042201 of GA CAS.

\section{References}

Bezrukikh, V. V., N. A. Barabanov, Yu. I. Venediktov, V. I. Zhdanov, V. I. Ivchenko, G. A. Kotova, L. A. Lezhen, S. A. Orzhinsky, and V. I. Prokhorenko, Investigation of low-energy plasma in the Earth's magnetosphere on board the Tail and Auroral probes: Instrumentation and preliminary results, Cosmic Research, 36, 30-38, 1998.

Carpenter, D. L. and R. R. Anderson, An ISEE/whistler model of equatorial electron density in the magnetosphere, J. Geophys. Res., 97, 1097-1108, 1992.

Carpenter, D. L. and C. R. Chappell, Satellite studies of magnetospheric substorms on August 15, 1968, 3. Some features of magnetospheric convection, J. Geophys. Res., 78, 3062-3067, 1973.

Carpenter, D. L. and C. G. Park, On what ionospheric workers should know about the plasmapause-plasmasphere, Rev. Geophys. Space Phys., 11(1), 133-154, 1973.

Carpenter, D. L., K. Stone, J. Siren, and T. L. Crystal, Magnetospheric electric fields deduced from drifting whistler paths, J. Geophys. Res., 77, 1219, 1972.

Carpenter, D. L., R. R. Anderson, W. Calvert, and M. B. Moldwin, CRRES observations of density cavities inside the plasmasphere, J. Geophys. Res., 105(A10), 23323-23338, 2000.

Chappell, C. R., Detached plasma regions in the magnetosphere, J. Geophys. Res., 79, 1861-1870, 1974.

Chen, A. J. and J. M. Grebowsky, Plasma tail interpretations of pronounced detached plasma regions measured by OGO 5, J. Geophys. Res., 79(25), 3851-3855, 1974.

Green, J. L., B. R. Sandel, S. F. Fung, D. L. Gallagher, and B. W. Reinisch, On the origin of kilometric continuum, J. Geophys. Res., 107(A7), 10.1029/2001JA000193, 2002.

Higel, B. and L. Wu, Electron density and plasmapause characteristics at 6.6 RE: a statistical study of the GEOS 2 relaxation sounder data, J. Geophys. Res., 89, 1583-1601, 1984.

Horwitz, J. L., R. H. Comfort, and C. R. Chappell, A statistical characterization of plasmasphere density structure and boundary locations, $J$. Geophys. Res., 95(A6), 7937-7947, 1990.

Kotova, G. A., V. V. Bezrukikh, M. I. Verigin, L. A. Lezhen, N. A. Barabanov, Interball 1 / alpha 3 cold plasma measurements in the evening plasmasphere: Quiet and disturbed magnetic conditions, Adv. Space Res., 30(10), 2313-2318, 2002a.

Kotova, G. A., V. V. Bezrukikh, M. I. Verigin, and L. A. Lezhen, Temperature and density variations in the dusk and dawn plsmasphere as observed by INTERBALL TAIL in 1999-2000, Adv. Space Res., 30(7), 1831-1834, 2002b.

Lemaire, J. F., The formation of plasmaspheric tails, Phys. Chem. Earth (C), 25(1-2), 9-17, 2000.

Lemaire, J. F. and K. I. Gringauz with contribution from D. L. Carpenter and V. Bassolo, The Earth's Plasmasphere, Cambridge University Press, 1998.

McIlwain, C. E., A Kp dependent equatorial electric field model, The physics of thermal plasma in the magnetosphere, Adv. Space Res., 6(3), 187-197, 1986.

Moldwin, M. B., L. Downward, H. K. Rassoul, R. Amin, R. R. Anderson, A new model of the location of the plasmapause: CRRES results, $J$. Geophys. Res., 107(A11), 1339, doi:10.1029/2001JA009211, 2002.

Pierrard, V. and J. F. Lemaire, Development of shoulders and plumes in the frame of the interchange instability mechanism for plasmapause formation, Geophys. Res. Lett., 31(5), L05809, 10.1029/2003GL018919, 2004.

Sandel, B. R., R. A. King, W. T. Forrester, D. L. Gallagher, A. L. Broadfoot, and C. C. Curtis, Initial results from the IMAGE Extreme Ultraviolet Imager, Geophys. Res. Lett., 28(8), 1439-1442, 2001.

Sandel, B. R., J. Goldstein, D. L. Gallagher, and M. Spasojevic, Extreme ultraviolet imager observations of the structure and dynamics of the plasmasphere, Space Sci. Rev., 109, 25-46, 2003.

G. Kotova (e-mail: kotova@iki.rssi.ru), V. Bezrukikh, M. Verigin, and J. Smilauer 